\title{
A NOTE ON INTERSECTION MULTIPLICITIES
}

Tor H. Gulliksen

Let $\mathrm{R}, \mathrm{M}$ denote a local (noetherian) ring and let $M$ and $N$ be R-modules such that $M \otimes N$ has finite length $I(M \otimes N)$. If $R$ is regular we can define the intersection multiplicity:

$$
x^{R}(M, N)=\Sigma_{i}(-1)^{i} I\left(\operatorname{Tor}_{i}^{R}(M, N)\right)
$$

as in Serre [2]. The purpose of this note is to prove the following theorem which shows that there is a natural way to extend the notion of intersection multiplicities to the theory of modules over arbitrary local rings $R$. The $R / m$ - vectorspace dimension of $M / m^{2}$ will be called the imbedding dimension of $R$.

THEOREM. Let $\mathrm{C}$ be an arbitrary local (noetherian) ring, and let $M$ and $N$ be $E$ - modules of finite type such $M \otimes N$ has finite length. Assume that $A \rightarrow C$ and $B \rightarrow C$ are surjective ringhomomoriphisms, $A$ and $B$ being regular local rings of minimal dimension, that is the dimension of $A$ and $B$ equals the imbedding dimension of C. Then

$$
\chi^{A}(M, N)=x^{B}(M, N) .
$$

PROOF. We may assume that $A, B$ and $C$ are complete local rings. Hence so is the fiber-product $A \times{ }_{C} B$. By Cohen's structure theorem $\mathrm{A} \times{ }_{\mathrm{c}} \mathrm{B}$ is a homomorphic image of a regular local ring $R$, thus we have a commutative diagram of surjective ringhomomorphisms

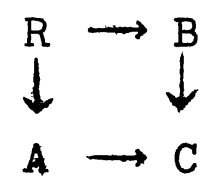


Put $a:=\operatorname{Ker}(R \rightarrow A)$ and $\not b:=\operatorname{Ker} \quad(R \rightarrow B)$. Let $M$ be the maximal ideal of $R$. Since $A, B$ and $R$ are all regular, the inclusions

$O Z \subset M$ and $b \subset M$ give rise to injections

$$
\begin{aligned}
& a / m a \rightarrow m / m^{2} \\
& b / m b \rightarrow m / m^{2}
\end{aligned}
$$

By means of these maps we will consider $a / m a$ and $b / m b$ as subspaces of $M / M^{2}$. Put

$$
S=\operatorname{dim} R-\operatorname{dim} A
$$

Since $\operatorname{dim} A=\operatorname{dim} B$ both $\alpha$ and $b$ are minimally generated by $s$ elements. Let $\partial_{1}, \ldots, \partial_{r}(r \leq s)$ be a basis for $a / m a n b / 4 b$. Let $a_{1}, \ldots, a_{r}$ respectively $b_{1}, \ldots, b_{r}$ be elements in $a$ respectively $\hbar$ representing $\partial_{1}, \ldots, \partial_{r^{\circ}}$. Now extend these two sequences to minimal sets of generators

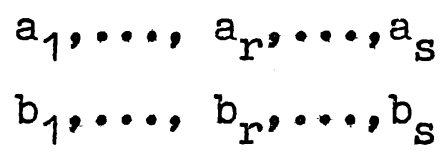

for $a$ and $b$ respectively. For each $i(0 \leq i \leq s)$ the elements

$$
a_{1}, \ldots, a_{i}, b_{i+1}, \ldots, b_{s}
$$

represent linearly independent elements in $M / m^{2}$. Hence they are part of a regular system of parameters for $R$. Let $\boldsymbol{G}_{i}$ denote the ideal they generate and put

$$
A_{i}:=R / \sigma_{i}
$$

Then each $A_{i}$ is a regular local ring. Observe that $A_{0}=B$ and $A_{S}=A$. In the following let $1 \leq i \leq s$. To prove the theorem it clearly suffices to prove

$$
x^{A_{i-1}}(M, N)=x^{A_{i}}(M, N)
$$


Here we will use a technique which was used in [1] for a similar purpose. To simplify the notation we put $P:=A_{i-1}$ and $Q:=A_{i}$. Let $I$ be the ring $R / \mathbb{C}$ where $\mathbb{C}$ is the ideal generated by

$$
a_{1}, \ldots, a_{i-1}, a_{i}, b_{i}, b_{i+1}, \ldots, b_{s}
$$

Observe that $L$ need not be regular. We have exact sequences:

$$
\begin{aligned}
& O \rightarrow P \stackrel{a_{i}}{\rightarrow} P \rightarrow L \rightarrow 0 \\
& O \rightarrow Q \stackrel{b_{i}}{\longrightarrow} Q \rightarrow L \rightarrow 0
\end{aligned}
$$

where $a_{i}$ and $b_{i}$ denotes multiplication by $a_{i}$ and $b_{i}$ respectively. From the sequences above we obtain standard spectralsequences

$$
\operatorname{Tor}_{p}^{L}\left(M, \operatorname{Tor}_{q}^{R}(N, L)\right) \Rightarrow \operatorname{Tor}_{p+q}^{P}(M, N)
$$

and

$$
\operatorname{Tor}_{p}^{L}\left(M, \operatorname{Tor}_{q}^{Q}(N, L)\right) \Rightarrow \operatorname{Tor}_{p+q}^{Q}(M, N)
$$

where $\operatorname{Tor}_{q}^{P}(N, L)$ and $\operatorname{Tor}_{q}^{Q}(N, L)$ equals $N$ for $q=0,1$ and eqnals zero for $q \neq 0,1$.

Hence we obtain exact sequences

$$
\begin{aligned}
& \ldots \operatorname{Tor}_{i}^{L}(M, N) \rightarrow \operatorname{Tor}_{i+1}^{P}(M, N) \rightarrow \operatorname{Tor}_{i+1}^{L}(M, N) \rightarrow \operatorname{Tor}_{i-1}^{L}(M, N) \rightarrow \ldots \\
& \ldots \operatorname{Tor}_{i}^{L}(M, N) \rightarrow \operatorname{Tor}_{i+1}^{Q}(M, N) \rightarrow \operatorname{Tor}_{i+1}^{L}(M, N) \rightarrow \operatorname{Tor}_{i-1}^{L}(M, N) \rightarrow \ldots
\end{aligned}
$$

from which it follows that

$$
x^{P}(M, N)=x^{Q}(M, N)
$$

\section{References}

1. M.-P. Malliavin - Brameret, Une remarque sur les anneaux locavx réguliers, Seminaire Dubreil - Pisot (Algèbre et Théorie des Nombres), 1970/1971 no.13.

2. J.P. Serre, Algèbre Locale Multiplicités, (Lecture Notes in Mathematics 11), Springer - Verlag, 1965. 